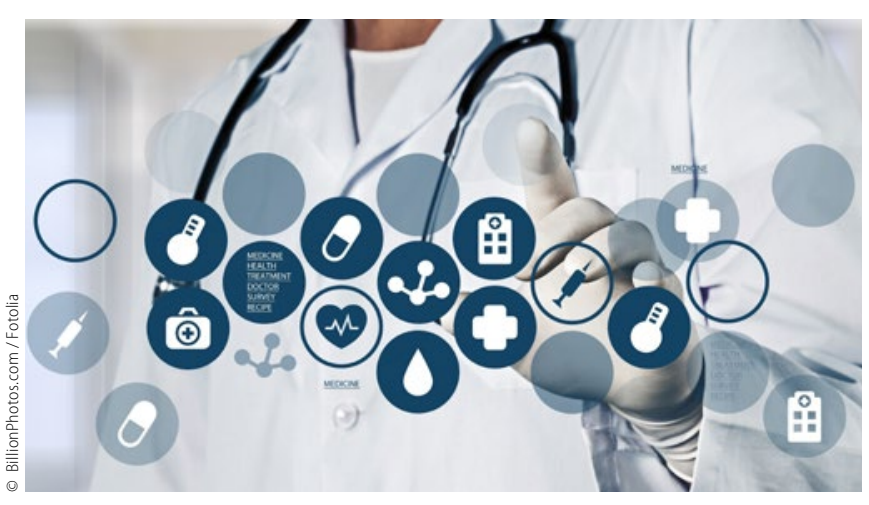

\section{Wenn's mal wieder länger dauert: Das E-Health-Gesetz}

Geht es um E-Health, rückt das vermeintliche Wohl der Patienten schnell in den Fokus. Zumindest werden Politiker nicht müde zu betonen, dass Telemedizin im Gesundheitswesen maßgebliche Vorteile für die Versorgung im ländlichen Raum hat. Und so ist es nur logisch, dass der Bundestag das E-Health-Gesetz („,Gesetz für sichere digitale Kommunikation und Anwendungen im Gesundheitswesen“) verabschiedete, das Anfang 2016 in Kraft getreten ist. Mit dem Gesetz sollen Informationsund Kommunikationstechnologien in der medizinischen Versorgung etabliert und die digitale Vernetzung vorangebracht werden. Ziel ist es, alle Akteure im Gesundheitswesen miteinander zu verbinden und Patientendaten schnell digital abrufbar zu machen. „Dabei stehen Patientennutzen und Datenschutz im Mittelpunkt", wirft Bundesgesundheitsminister Hermann Gröhe (CDU) immer wieder gerne ein. Datenschützer hingegen zweifeln an der Sicherheit der Patientendaten, wenn diese erstmal tagtäglich durchs weltweite Netz gejagt werden.

Das E-Health-Gesetz enthält Vorgaben für die Telematikinfrastruktur (TI), feste Termine für die Umsetzung, Anreize für Ärzte und Zahnärzte sowie Sanktionen. Doch was die Termine betrifft, ist der Zeitplan für den Aufbau der TI immer wieder durcheinander geraten. Eigentlich sollte die elektronische Prüfung und Aktualisierung von Versichertenstammdaten nach einer Erprobungsphase ab dem 1. Juli 2016 innerhalb von zwei Jahren flächendeckend eingeführt werden. Aber die Industrie hatte Probleme mit der Lieferung der Kartenlesegeräte und Konnektoren, die in den Praxen für eine sichere Verbindung zur IT sorgen sollen.

Laut E-Health-Gesetz drohen den Spitzenorganisationen der Leistungserbringer und Kostenträger weiterhin Kürzungen der Haushaltsmittel, wenn die gesetzlich festgeschriebenen Fristen nicht eingehalten werden. Ärzte und Zahnärzte müssen mit Vergütungsabschlägen rechnen. Doch ohne entsprechende Geräte funktioniert es leider nicht. Für Minister Gröhe kein Grund, nicht weiter auf die Tube zu drücken. Erst im November betonte er erneut, das Tempo bei der Digitalisierung im Gesundheitswesen voranzutreiben. Und just zur selben Zeit kam denn auch die für ihn erfreuliche Nachricht: Nach einem Bericht der „Frankfurter Allgemeine Zeitung“(FAZ) sollen die ersten Arztpraxen die Verschlüsselungsgeräte für den OnlineRollout der elektronischen Gesundheitskarte (eGK) erhalten haben. Die $F A Z$ berief sich auf Informationen der Gesellschaft für Telematikanwendungen der Gesundheitskarte (Gematik). Und die sollte es wissen.

\section{Schulterschluss: Zahnärzte erarbeiten PAR-Konzept}

Vertreter zahnärztlicher Organisationen sind sich einig: Auch die 50 Jahre alten Richtlinien der geltenden Parodontitistherapie (PAR) widersprechen der medizinisch-technischen Entwicklung und müssen modernisiert werden. Deshalb hat die Zahnärzteschaft in diesem Jahr damit begonnen, gemeinsam ein Konzept für PAR in der gesetzlichen Krankenversicherung (GKV) zu erarbeiten. Kassenzahnärztliche Bundesvereinigung (KZBV), Bundeszahnärztekammer (BZÄK) und der Freie Verband Deutscher Zahnärzte (FVDZ) ziehen demonstrativ an einem Strang. Oberstes Ziel ist es, die Mittel der GKV zielgerichtet für notwendige Veränderungen des Versorgungskatalogs einzusetzen. Das Ergebnis soll dann in den Gemeinsamen Bundesausschuss (G-BA) eingebracht werden.

KZBV, BZÄK, FVDZ und auch die Wissenschaft sind sich einig, dass eine moderne Parodontitistherapie unverzichtbar und alternativlos ist und präventionsorientiert sein muss. Am runden Tisch wird ein geeignetes Behandlungskonzept ausgearbeitet. Dass dies nötig ist, belegen aktuelle Zahlen. Auf der FVDZHauptversammlung sprach der Parodontologe Professor Dr. Christof Dörfer von insgesamt elf Millionen Menschen in Deutschland, die unter Parodontitis leiden. Als ein Problem bezeichnete der Experte von der Universität in Kiel die mangelhafte Früherkennung. Die nämlich sei der Schlüssel für eine erfolgreiche PAR-Therapie. Die Zahnärzteschaft will jetzt Finanzierungskonzepte erarbeiten. Dazu gehört auch die Diskussion, ob und wie weit eine unterstützende PAR-Therapie (UPT) in der GKV verankert werden kann, und die entscheidende Frage, was die Krankenkassen bereit sind zu zahlen. Die Körperschaften wollen den Sachleistungskatalog jedenfalls nicht aufblättern. Was die Voraussetzungen an eine Parodontalbehandlung in der GKV betrifft, hat der Freie Verband verschiedene Grundsätze festgelegt, die auch alle Erwartungen der KZBV erfüllen und bei einer Reform der PAR-Therapie eingehalten werden sollen. Dazu gehört beispielsweise die Forderung, dass die Eigenverantwortung des Patienten für einen Therapieerfolg sichergestellt wird. Auch ist die Bewertung der zahnärztlichen PAR-Leistungen aus Sicht des Freien Verbandes betriebswirtschaftlich so zu kalkulieren, dass sie von Zahnärzten erbracht werden können. Und nicht zuletzt müssen für zusätzliche Leistungen auch zusätzliche Mittel bereitgestellt werden.

$m f$

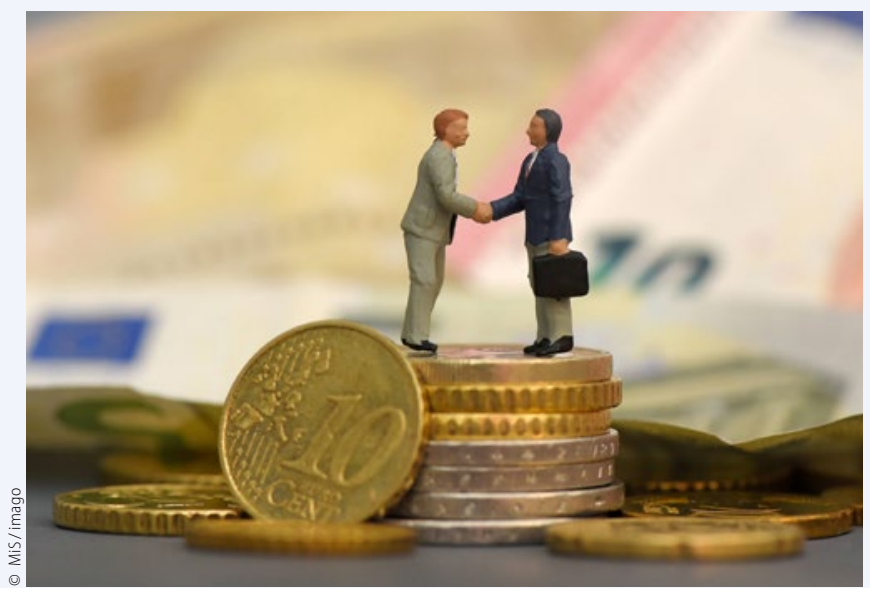

